\title{
Bounds for Orders of Derivatives in Differential Elimination Algorithms
}

\author{
Richard Gustavson \\ CUNY Graduate Center \\ Ph.D. Program in Mathematics \\ 365 Fifth Avenue \\ New York, NY 10016, USA \\ rgustavson@gradcenter. \\ cuny.edu
}

\author{
Alexey Ovchinnikov \\ CUNY Queens College \\ Department of Mathematics \\ 65-30 Kissena Blvd \\ Queens, NY 11367-1597, USA \\ aovchinnikov@qc.cuny.edu pogudin.gleb@gmail.com \\ Gleb Pogudin \\ Moscow State University \\ Department of Mechanics and \\ Mathematics \\ Moscow, 119991, Russia
}

\begin{abstract}
We compute an upper bound for the orders of derivatives in the Rosenfeld-Gröbner algorithm. This algorithm computes a regular decomposition of a radical differential ideal in the ring of differential polynomials over a differential field of characteristic zero with an arbitrary number of commuting derivations. This decomposition can then be used to test for membership in the given radical differential ideal. In particular, this algorithm allows us to determine whether a system of polynomial PDEs is consistent.

Previously, the only known order upper bound was given by Golubitsky, Kondratieva, Moreno Maza, and Ovchinnikov for the case of a single derivation. We achieve our bound by associating to the algorithm antichain sequences whose lengths can be bounded using the results of León Sánchez and Ovchinnikov.
\end{abstract}

\section{CCS Concepts}

-Computing methodologies $\rightarrow$ Symbolic and algebraic algorithms; Algebraic algorithms;

\section{Keywords}

Polynomial differential equations; differential elimination algorithms; computational complexity

\section{INTRODUCTION}

The Rosenfeld-Gröbner algorithm is a fundamental algorithm in the algebraic theory of differential equations. This algorithm, which first appeared in $[1,2]$, takes as its input a finite set $F$ of differential polynomials and outputs a representation of the radical differential ideal generated by $F$ as a finite intersection of regular differential ideals. The Rosenfeld-Gröbner algorithm has many applications; for example, it can be used to test membership in a radical differential ideal, and, in conjunction with the differential Null-

Permission to make digital or hard copies of all or part of this work for personal or classroom use is granted without fee provided that copies are not made or distributed for profit or commercial advantage and that copies bear this notice and the full citation on the first page. Copyrights for components of this work owned by others than ACM must be honored. Abstracting with credit is permitted. To copy otherwise, or republish, to post on servers or to redistribute to lists, requires prior specific permission and/or a fee. Request permissions from permissions@ acm.org.

ISSAC '16, July 19-22, 2016, Waterloo, ON, Canada

(c) 2016 ACM. ISBN 978-1-4503-4380-0/16/07 . . \$15.00

DOI: http://dx.doi.org/10.1145/2930889.2930922 stellensatz, can be used to test the consistency of a system of polynomial differential equations. See [6] for a history of the development of the Rosenfeld-Gröbner algorithm and similar decomposition algorithms.

The Rosenfeld-Gröbner algorithm has been implemented in MAPLE as a part of the DifferentialAlgebra package. In order to determine the complexity of the algorithm, we need to (among other things) find an upper bound on the orders of derivatives that appear in all intermediate steps and in the output of the algorithm. The first step in answering this question was completed in [6], in which an upper bound in the case of a single derivation and any ranking on the set of derivatives was found. If there are $n$ differential indeterminates and the order of the original system is $h$, the authors showed that an upper bound on the orders of the output of the Rosenfeld-Gröbner algorithm is $h(n-1)$ !.

In this paper, we extend this result by finding an upper bound for the orders of derivatives that appear in the intermediate steps and in the output of the Rosenfeld-Gröbner algorithm in the case of an arbitrary number of commuting derivations and an orderly ranking on the derivatives. Our result depends on studying lengths of antichain sequences in the set $\mathbb{Z}_{\geqslant 0}^{m} \times\{1, \ldots, n\}$ equipped with a specific partial order. This analysis began in [12] and continued in [4, 11].

We show that an upper bound for the orders of derivatives in the intermediate steps and in the output of the RosenfeldGröbner algorithm is given by $h f_{L+1}$, where $h$ is the order of our input system of differential equations, $\left\{f_{0}, f_{1}, f_{2}, \ldots\right\}$ is the Fibonacci sequence $\{0,1,1,2,3,5, \ldots\}$, and $L$ is the maximal possible length of a certain antichain sequence (that depends solely on $h$, the number of derivations, and the number of differential indeterminates). Note that this is different from the upper bounds for the effective differential Nullstellensatz [3, 8], which are higher and also depend on the degree of the given system of differential equations.

The paper is organized as follows. In Section 2, we present the background material from differential algebra that is necessary to understand the Rosenfeld-Gröbner algorithm. In Section 3, we describe this algorithm as it is presented in [9], as well as two necessary auxiliary algorithms. In Section 4 , we prove our main result on the upper bound. In Section 5, we calculate the upper bound for specific values using the results of [11]. In Section 6, we give an example showing that the lower bound for the orders of derivatives in the Rosenfeld-Gröbner algorithm is at least double-exponential in the number of derivations. 


\section{BACKGROUND ON DIFFERENTIAL ALGEBRA}

In this section, we present background material from differential algebra that is pertinent to the Rosenfeld-Gröbner algorithm. For a more in-depth discussion, we turn the reader to $[9,10]$.

Definition 1. A differential ring is a commutative ring $R$ with a collection of $m$ commuting derivations $\Delta=$ $\left\{\partial_{1}, \ldots, \partial_{m}\right\}$ on $R$.

Definition 2. An ideal $I$ of a differential ring is a differential ideal if $\delta a \in I$ for all $a \in I$ and $\delta \in \Delta$.

For a set $A \subseteq R$, we let $(A), \sqrt{(A)},[A]$, and $\{A\}$ denote the smallest ideal, radical ideal, differential ideal, and radical differential ideal containing $A$, respectively. If $\mathbb{Q} \subseteq R$, then $\{A\}=\sqrt{[A]}$.

Remark 1. In this paper, as usual, we also use the braces $\left\{a_{1}, a_{2}, \ldots\right\}$ to denote the set containing the elements $a_{1}, a_{2}, \ldots$ Even though this notation conflicts with the above notation for radical differential ideals (used here for historical reasons), it will be clear from the context which of the two objects we mean in each particular situation.

In this paper, $\mathbf{k}$ is a differential field of characteristic zero with $m$ commuting derivations. The set of derivative operators is denoted by

$$
\Theta:=\left\{\partial_{1}^{i_{1}} \cdots \partial_{m}^{i_{m}}: i_{j} \in \mathbb{Z}_{\geqslant 0}, 1 \leqslant j \leqslant m\right\} .
$$

For $Y=\left\{y_{1}, \ldots, y_{n}\right\}$ a set of $n$ differential indeterminates, the set of derivatives of $Y$ is

$$
\Theta Y:=\{\theta y: \theta \in \Theta, y \in Y\} .
$$

Then the ring of differential polynomials over $\mathbf{k}$ is defined to be

$$
\mathbf{k}\{Y\}=\mathbf{k}\left\{y_{1}, \ldots, y_{n}\right\}:=\mathbf{k}[\theta y: \theta y \in \Theta Y] .
$$

We can naturally extend the derivations $\partial_{1}, \ldots, \partial_{m}$ to the ring $\mathbf{k}\{Y\}$ by defining

$$
\partial_{j}\left(\partial_{1}^{i_{1}} \cdots \partial_{m}^{i_{m}} y_{k}\right):=\partial_{1}^{i_{1}} \cdots \partial_{j}^{i_{j}+1} \cdots \partial_{m}^{i_{m}} y_{k}
$$

For any $\theta=\partial_{1}^{i_{1}} \cdots \partial_{m}^{i_{m}} \in \Theta$, we define the order of $\theta$ to be

$$
\operatorname{ord}(\theta):=i_{1}+\cdots+i_{m} .
$$

For any derivative $u=\theta y \in \Theta Y$, we define

$$
\operatorname{ord}(u):=\operatorname{ord}(\theta) \text {. }
$$

For a differential polynomial $f \in \mathbf{k}\{Y\} \backslash \mathbf{k}$, we define the order of $f$ to be the maximum order of all derivatives that appear in $f$. For any finite set $A \subseteq \mathbf{k}\{Y\} \backslash \mathbf{k}$, we set

$$
\mathcal{H}(A):=\max \{\operatorname{ord}(f): f \in A\} .
$$

Definition 3. An orderly ranking on the set $\Theta Y$ is a total order $<$ satisfying the following three additional properties: for all $u, v \in \Theta Y$ and all $\theta \in \Theta, \theta \neq \mathrm{id}$,

1. $u<\theta u$

2. if $u<v$, then $\theta u<\theta v$
3. if $\operatorname{ord}(u)<\operatorname{ord}(v)$, then $u<v$.

For a derivative $u \in \Theta Y$, we let $(\Theta Y)_{<u}$ (respectively, $(\Theta Y)_{\leqslant u}$ ) be the collection of all derivatives $v \in \Theta Y$ with $v<u$ (respectively, $v \leqslant u$ ).

From now on, we fix an orderly ranking.

Definition 4. Let $f \in \mathbf{k}\{Y\} \backslash \mathbf{k}$.

- The derivative $u \in \Theta Y$ of highest rank appearing in $f$ is called the leader of $f$, denoted lead $(f)$.

- If we write $f$ as a univariate polynomial in $\operatorname{lead}(f)$, the leading coefficient is called the initial of $f$, denoted $\operatorname{init}(f)$.

- If we apply any derivative $\delta \in \Delta$ to $f$, the leader of $\delta f$ is $\delta(\operatorname{lead}(f))$, and the initial of $\delta f$ is called the separant of $f$, denoted $\operatorname{sep}(f)$.

Given a set $A \subseteq \mathbf{k}\{Y\} \backslash \mathbf{k}$, we will denote the set of leaders of $A$ by $\mathfrak{L}(A)$, the set of initials of $A$ by $I_{A}$, and the set of separants of $A$ by $S_{A}$; we then let $H_{A}=I_{A} \cup S_{A}$ be the set of initials and separants of $A$. For any derivative $u \in \Theta Y$, we let $A_{<u}$ (respectively, $A_{\leqslant u}$ ) be the elements of $A$ with leader $<u$ (respectively, $\leqslant u$ ), that is,

$$
A_{<u}:=A \cap \mathbf{k}\left[(\Theta Y)_{<u}\right] \quad \text { and } \quad A_{\leqslant u}:=A \cap \mathbf{k}\left[(\Theta Y)_{\leqslant u}\right] .
$$

We can similarly define $(\Theta A)_{<u}$ and $(\Theta A)_{\leqslant u}$, where

$$
\Theta A:=\{\theta f: \theta \in \Theta, f \in A\} .
$$

Given $f \in \mathbf{k}\{Y\} \backslash \mathbf{k}$ such that $\operatorname{deg}_{\text {lead }(f)}(f)=d$, we define the rank of $f$ to be

$$
\operatorname{rank}(f):=\operatorname{lead}(f)^{d} .
$$

The orderly ranking $<$ on $\Theta Y$ determines a pre-order (that is, a relation satisfying all of the properties of an order, except for the property that $a \leqslant b$ and $b \leqslant a$ imply that $a=b)$ on $\mathbf{k}\{Y\} \backslash \mathbf{k}$, as follows.

Definition 5. Given $f_{1}, f_{2} \in \mathbf{k}\{Y\} \backslash \mathbf{k}$ such that $\operatorname{deg}_{\text {lead }\left(f_{1}\right)}\left(f_{1}\right)=d_{1}$ and $\operatorname{deg}_{\text {lead }\left(f_{2}\right)}\left(f_{2}\right)=d_{2}$, we say that

$$
\operatorname{rank}\left(f_{1}\right)<\operatorname{rank}\left(f_{2}\right)
$$

if lead $\left(f_{1}\right)<\operatorname{lead}\left(f_{2}\right)$ or if lead $\left(f_{1}\right)=\operatorname{lead}\left(f_{2}\right)$ and $d_{1}<d_{2}$.

Definition 6. A differential polynomial $f$ is partially reduced with respect to another differential polynomial $g$ if no proper derivative of lead $(g)$ appears in $f$, and $f$ is reduced with respect to $g$ if, in addition,

$$
\operatorname{deg}_{\text {lead }(g)}(f)<\operatorname{deg}_{\text {lead }(g)}(g) .
$$

A differential polynomial is then (partially) reduced with respect to a set $A \subseteq \mathbf{k}\{Y\} \backslash \mathbf{k}$ if it is (partially) reduced with respect to every element of $A$.

Definition \%. For a set $A \subseteq \mathbf{k}\{Y\} \backslash \mathbf{k}$, we say that $A$ is:

- autoreduced if every element of $A$ is reduced with respect to every other element.

- weak d-triangular if $\mathfrak{L}(A)$ is autoreduced.

- $d$-triangular if $A$ is weak d-triangular and every element of $A$ is partially reduced with respect to every other element. 
Note that every autoreduced set is d-triangular. Every weak d-triangular set (and thus every d-triangular and autoreduced set) is finite (see [9, Proposition 3.9]). Since the set of leaders of a weak d-triangular set $A$ is autoreduced, distinct elements of $A$ must have distinct leaders. If $u \in \Theta Y$ is the leader of some element of a weak d-triangular set $A$, we let $A_{u}$ denote this element.

Definition 8. We define a pre-order on the collection of all weak d-triangular sets, which we also call rank, as follows. Given two weak d-triangular sets $A=\left\{A_{1}, \ldots, A_{r}\right\}$ and $B=\left\{B_{1}, \ldots, B_{s}\right\}$, in each case arranged in increasing rank, we say that $\operatorname{rank}(A)<\operatorname{rank}(B)$ if either:

- there exists a $k \leqslant \min (r, s)$ such that $\operatorname{rank}\left(A_{i}\right)=$ $\operatorname{rank}\left(B_{i}\right)$ for all $1 \leqslant i<k$ and $\operatorname{rank}\left(A_{k}\right)<\operatorname{rank}\left(B_{k}\right)$, or

- $r>s$ and $\operatorname{rank}\left(A_{i}\right)=\operatorname{rank}\left(B_{i}\right)$ for all $1 \leqslant i \leqslant s$.

We also say that $\operatorname{rank}(A)=\operatorname{rank}(B)$ if $r=s$ and $\operatorname{rank}\left(A_{i}\right)=$ $\operatorname{rank}\left(B_{i}\right)$ for all $1 \leqslant i \leqslant r$.

We can restrict this ranking to the collection of all dtriangular sets or the collection of all autoreduced sets.

Definition 9. A characteristic set of a differential ideal $I$ is an autoreduced set $C \subseteq I$ of minimal rank among all autoreduced subsets of $I$.

Given a finite set $S \subseteq \mathbf{k}\{Y\}$, denote by $S^{\infty}$ the multiplicative set containing 1 and generated by $S$. For an ideal $I \subseteq \mathbf{k}\{Y\}$, we define the colon ideal to be

$$
I: S^{\infty}:=\left\{a \in \mathbf{k}\{Y\}: \exists s \in S^{\infty} \text { with } s a \in I\right\} .
$$

If $I$ is a differential ideal, then $I: S^{\infty}$ is also a differential ideal (see [10, Chapter I, Section 2]).

Definition 10. Given a differential polynomial $f \in \mathbf{k}\{Y\}$ and a weak d-triangular set $A \subseteq \mathbf{k}\{Y\}$, a differential partial remainder $f_{1}$ and a differential remainder $f_{2}$ of $f$ with respect to $A$ are differential polynomials such that there exist $s \in S_{A}^{\infty}$ and $h \in H_{A}^{\infty}$ such that $s f \equiv f_{1} \bmod [A]$ and $h f \equiv f_{2} \bmod [A]$, with $f_{1}$ partially reduced with respect to $A$ and $f_{2}$ reduced with respect to $A$.

We denote a differential partial remainder of $f$ with respect to $A$ by pd-red $(f, A)$ and a differential remainder of $f$ with respect to $A$ by d-red $(f, A)$. There are algorithms to compute pd-red $(f, A)$ and d-red $(f, A)$ for any $f$ and $A$ (see [9, Algorithm $3.12 \&$ Algorithm 3.13]). These algorithms have the property that

$$
\operatorname{rank}(\operatorname{pd}-\operatorname{red}(f, A)), \operatorname{rank}(\mathrm{d}-\operatorname{red}(f, A)) \leqslant \operatorname{rank}(f) ;
$$

since we have an orderly ranking, this implies that

$$
\operatorname{ord}(\operatorname{pd}-\operatorname{red}(f, A)), \operatorname{ord}(\mathrm{d}-\operatorname{red}(f, A)) \leqslant \operatorname{ord}(f) .
$$

Definition 11. Two derivatives $u, v \in \Theta Y$ are said to have a common derivative if there exist $\phi, \psi \in \Theta$ such that $\phi u=$ $\psi v$. Note this is the case precisely when $u=\theta_{1} y$ and $v=\theta_{2} y$ for some $y \in Y$ and $\theta_{1}, \theta_{2} \in \Theta$.

Definition 12. If $u=\partial_{1}^{i_{1}} \cdots \partial_{m}^{i_{m}} y$ and $v=\partial_{1}^{j_{1}} \cdots \partial_{m}^{j_{m}} y$ for some $y \in Y$, we define the least common derivative of $u$ and $v$, denoted $\operatorname{lcd}(u, v)$, to be

$$
\operatorname{lcd}(u, v)=\partial_{1}^{\max \left(i_{1}, j_{1}\right)} \cdots \partial_{m}^{\max \left(i_{m}, j_{m}\right)} y .
$$

Definition 13. For $f, g \in \mathbf{k}\{Y\} \backslash \mathbf{k}$, we define the $\Delta$ polynomial of $f$ and $g$, denoted $\Delta(f, g)$, as follows. If lead $(f)$ and lead $(g)$ have no common derivatives, set $\Delta(f, g)=0$. Otherwise, let $\phi, \psi \in \Theta$ be such that

$$
\operatorname{lcd}(\operatorname{lead}(f), \operatorname{lead}(g))=\phi(\operatorname{lead}(f))=\psi(\operatorname{lead}(g)),
$$

and define

$$
\Delta(f, g):=\operatorname{sep}(g) \phi(f)-\operatorname{sep}(f) \psi(g)
$$

Definition 14. A pair $(A, H)$ is called a regular differential system if the following four conditions hold:

- $A$ is a d-triangular set

- $H$ is a set of differential polynomials that are all partially reduced with respect to $A$

- $S_{A} \subseteq H^{\infty}$

- for all $f, g \in A$,

$$
\Delta(f, g) \in\left((\Theta A)_{<u}\right): H^{\infty},
$$

where $u=\operatorname{lcd}(\operatorname{lead}(f), \operatorname{lead}(g))$.

Definition 15. Any ideal of the form $[A]: H^{\infty}$, where $(A, H)$ is a regular differential system, is called a regular differential ideal.

Every regular differential ideal is a radical differential ideal (see [9, Theorem 4.12]).

Definition 16. Given a radical differential ideal $I \subseteq$ $\mathbf{k}\{Y\}$, a regular decomposition of $I$ is a finite collection of regular differential systems $\left\{\left(A_{1}, H_{1}\right), \ldots,\left(A_{r}, H_{r}\right)\right\}$ such that

$$
I=\bigcap_{i=1}^{r}\left[A_{i}\right]: H_{i}^{\infty} .
$$

Due to the Rosenfeld-Gröbner algorithm, every radical differential ideal in $\mathbf{k}\{Y\}$ has a regular decomposition.

Definition 17. A d-triangular set $C$ is called a differential regular chain if it is a characteristic set of $[C]: H_{C}^{\infty}$; in this case, we call $[C]: H_{C}^{\infty}$ a characterizable differential ideal.

Definition 18. A characteristic decomposition of a radical differential ideal $I \subseteq \mathbf{k}\{Y\}$ is a representation of $I$ as an intersection of characterizable differential ideals.

As we will recall in Section 3, every radical differential ideal also has a characteristic decomposition.

\section{ROSENFELD-GRÖBNER ALGORITHM}

Below we reproduce the Rosenfeld-Gröbner algorithm from [9, Section 6]. This algorithm relies on two others, called auto-partial-reduce and update, which we also include. We need to include these two auxiliary algorithms because, in Section 4, we will study their effect on the growth of the orders of derivatives in the Rosenfeld-Gröbner algorithm. 
The Rosenfeld-Gröbner algorithm takes as its input two finite subsets $F, K \in \mathbf{k}\{Y\}$ and outputs a finite set $\mathcal{A}$ of regular differential systems such that

$$
\{F\}: K^{\infty}=\bigcap_{(A, H) \in \mathcal{A}}[A]: H^{\infty},
$$

where $\mathcal{A}=\varnothing$ if $1 \in\{F\}: K^{\infty}$.

If we have a decomposition of $\{F\}: K^{\infty}$ as in (2), we can compute, using only algebraic operations, a decomposition of the form

$$
\{F\}: K^{\infty}=\bigcap_{C \in \mathcal{C}}[C]: H_{C}^{\infty},
$$

where $\mathcal{C}$ is finite and each $C \in \mathcal{C}$ is a differential regular chain (see [9, Algorithm $7.1 \&$ Algorithm 7.2]). This means that an upper bound on $\bigcup_{(A, H) \in \mathcal{A}} \mathcal{H}(A \cup H)$ from (2) will also be an upper bound on $\bigcup_{C \in \mathcal{C}} \mathcal{H}(C)$ from (3).

The Rosenfeld-Gröbner algorithm has many immediate applications. For example, if $K=\{1\}$, then $\{F\}: K^{\infty}=$ $\{F\}$, so in this case, the Rosenfeld-Gröbner algorithm computes a regular decomposition of $\{F\}$, which then also gives us a characteristic decomposition of $\{F\}$ by the discussion in the previous paragraph.

The weak differential Nullstellensatz says that a system of polynomial differential equations $F=0$ is consistent (that is, has a solution in some differential field extension of $\mathbf{k}$ ) if and only if $1 \notin[F]$ (see [10, Chapter IV, Section 2]). Thus, since Rosenfeld-Gröbner $(F, K)=\varnothing$ if and only if $1 \in$ $\{F\}: K^{\infty}$, we see that $F=0$ is consistent if and only if Rosenfeld-Gröbner $(F,\{1\}) \neq \varnothing$.

More generally, the Rosenfeld-Gröbner algorithm and its extension for computing a characteristic decomposition of a radical differential ideal allow us to test for membership in a radical differential ideal, as follows. Suppose we have computed a characteristic decomposition

$$
\{F\}=\bigcap_{C \in \mathcal{C}}[C]: H_{C}^{\infty} .
$$

Now, a differential polynomial $f \in \mathbf{k}\{Y\}$ is contained in $\{F\}$ if and only if $f \in[C]: H_{C}^{\infty}$ for all $C \in \mathcal{C}$; this latter case is true if and only if d-red $(f, C)=0$, which can be tested using [9, Algorithm 3.13].

The Rosenfeld-Gröbner algorithm and its two auxiliary algorithms auto-partial-reduce and update rely on the following tuples of differential polynomials:

Definition 19. A Rosenfeld-Gröbner quadruple (or $R G$ quadruple) is a 4-tuple $(G, D, A, H)$ of finite subsets of $\mathbf{k}\{Y\}$ such that:

- $A$ is a weak d-triangular set

- $H_{A} \subseteq H$

- $D$ is a set of $\Delta$-polynomials

- for all $f, g \in A$, either $\Delta(f, g)=0$ or $\Delta(f, g) \in D$ or

$$
\Delta(f, g) \in\left(\Theta(A \cup G)_{<u}\right): H_{u}^{\infty},
$$

where $u=\operatorname{lcd}(\operatorname{lead}(f), \operatorname{lead}(g))$ and

$$
H_{u}=H_{A_{<u}} \cup\left(H \backslash H_{A}\right) \cap \mathbf{k}\left[(\Theta Y)_{<u}\right] .
$$

Remark 2. The RG-quadruple that is output by the update algorithm satisfies additional properties that we do not list, as they are not important for our analysis. For more information, we refer the reader to [9, Algorithm 6.10]
Algorithm: Rosenfeld-Gröbner, [9, Algorithm 6.11]

Data: $F, K$ finite subsets of $\mathbf{k}\{Y\}$

Result: A set $\mathcal{A}$ of regular differential systems such that:

- $\mathcal{A}$ is empty if it has been detected that $1 \in\{F\}: K^{\infty}$

- $\{F\}: K^{\infty}=\bigcap_{(A, H) \in \mathcal{A}}[A]: H^{\infty}$ otherwise

$\mathcal{S}:=\{(F, \varnothing, \varnothing, K)\}$

$\mathcal{A}:=\varnothing$

while $\mathcal{S} \neq \varnothing$ do

$(G, D, A, H):=$ an element of $\mathcal{S}$;

$\overline{\mathcal{S}}=\mathcal{S} \backslash(G, D, A, H)$;

if $G \cup D=\varnothing$ then

$\mathcal{A}:=\mathcal{A} \cup$ auto-partial-reduce $(A, H)$;

else

$p:=$ an element of $G \cup D$;

$\bar{G}, \bar{D}:=G \backslash\{p\}, D \backslash\{p\}$;

$\bar{p}:=\mathrm{d}-\operatorname{red}(p, A)$;

if $\bar{p}=0$ then

$\overline{\mathcal{S}}:=\overline{\mathcal{S}} \cup\{(\bar{G}, \bar{D}, A, H)\} ;$

else

if $\bar{p} \notin k$ then

$\bar{p}_{i}:=\bar{p}-\operatorname{init}(\bar{p}) \operatorname{rank}(\bar{p})$

$\bar{p}_{s}:=\operatorname{deg}_{\operatorname{lead}(\bar{p})}(\bar{p}) \bar{p}-\operatorname{lead}(\bar{p}) \operatorname{sep}(\bar{p}) ;$

$\overline{\mathcal{S}}:=\overline{\mathcal{S}} \cup\{$ update $(\bar{G}, \bar{D}, A, H, \bar{p}),(G \cup$

$\left.\left\{\bar{p}_{s}, \operatorname{sep}(\bar{p})\right\}, \bar{D}, A, H \cup\{\operatorname{init}(\bar{p})\}\right),(\bar{G} \cup$

$\left.\left.\left\{\bar{p}_{i}, \operatorname{init}(\bar{p})\right\}, \bar{D}, A, H\right)\right\}$;

end

end

end

$\mathcal{S}:=\overline{\mathcal{S}}$

end

return $\mathcal{A}$;

Algorithm: auto-partial-reduce, [9, Algorithm 6.8]

Data: Two finite subsets $A, H$ of $\mathbf{k}\{Y\}$ such that

Result:

$(\varnothing, \varnothing, A, H)$ is an RG-quadruple

- The empty set if it is detected that $1 \in[A]: H^{\infty}$

- Otherwise, a set with a single regular differential system $(B, K)$ with $\mathfrak{L}(A)=\mathfrak{L}(B), H_{B} \subseteq K$, and $[A]: H^{\infty}=[B]: K^{\infty}$

$B:=\varnothing$;

for $u \in \mathfrak{L}(A)$ increasingly do

$b:=\operatorname{pd}-\operatorname{red}\left(A_{u}, B\right)$

if $\operatorname{rank}(b)=\operatorname{rank}\left(A_{u}\right)$ then

$B:=B \cup\{b\}$;

else

| return $(\varnothing)$

end

end

$K:=H_{B} \cup\left\{\operatorname{pd}-\operatorname{red}(p, B): p \in H \backslash H_{A}\right\} ;$

if $0 \in K$ then

return $(\varnothing)$;

else

| return $\{(B, K)\}$;

end 
Algorithm: update, [9, Algorithm 6.10]

Data:

- A 4-tuple $(G, D, A, H)$ of finite subsets of $\mathbf{k}\{Y\}$

- A differential polynomial $p$ reduced with respect to $A$ such that $(G \cup\{p\}, D, A, H)$ is an RG-quadruple

Result: A new RG-quadruple $(\bar{G}, \bar{D}, \bar{A}, \bar{H})$

$u:=\operatorname{lead}(p)$;

$G_{A}:=\{a \in A \mid \operatorname{lead}(a) \in \Theta u\}$

$\bar{A}:=A \backslash G_{A}$;

$\bar{G}:=G \cup G_{A}$;

$\bar{D}:=D \cup\{\Delta(p, a) \mid a \in \bar{A}\} \backslash\{0\} ;$

$\bar{H}:=H \cup\{\operatorname{sep}(p), \operatorname{init}(p)\}$;

return $(\bar{G}, \bar{D}, \bar{A} \cup\{p\}, \bar{H})$;

\section{ORDER UPPER BOUND}

Given finite subsets $F, K \subseteq \mathbf{k}\{Y\}$, let $h=\mathcal{H}(F \cup K)$ (see (1)). Our goal is to find an upper bound for

$$
\mathcal{H}\left(\bigcup_{(A, H) \in \mathcal{A}}(A \cup H)\right),
$$

where $\mathcal{A}=\operatorname{Rosenfeld-\operatorname {Gröbner}}(F, K)$, in terms of $h, m$ (the number of derivations), and $n$ (the number of differential indeterminates).

We approach this problem as follows. Every $(A, H) \in \mathcal{A}$ is formed by applying the algorithm auto-partial-reduce to a 4-tuple $\left(\varnothing, \varnothing, A^{\prime}, H^{\prime}\right) \in \mathcal{S}$. Thus, it suffices:

- to bound how auto-partial-reduce increases the order of a collection of differential polynomials (it turns out to not increase the order), and

- to bound $\mathcal{H}(G \cup D \cup A \cup H)$ for all $(G, D, A, H)$ added to $\mathcal{S}$ throughout the course of the Rosenfeld-Gröbner algorithm.

We accomplish the latter by determining when the order of a tuple $(G, D, A, H)$ added to $\mathcal{S}$ is larger than the orders of the previous elements of $\mathcal{S}$ and bounding $\mathcal{H}(G \cup D \cup A \cup H)$ in this instance, and then bounding the number of times we can add such elements to $\mathcal{S}$.

There is a sequence $\left\{\left(G_{i}, D_{i}, A_{i}, H_{i}\right)\right\}_{i=0}^{N}$ corresponding to each regular differential system $(A, H)$ in the output of the Rosenfeld-Gröbner algorithm, where $N=N_{(A, H)}$, such that $\left(G_{i+1}, D_{i+1}, A_{i+1}, H_{i+1}\right)$ is obtained from $\left(G_{i}, D_{i}, A_{i}, H_{i}\right)$ during the while loop, $\left(G_{0}, D_{0}, A_{0}, H_{0}\right)=(F, \varnothing, \varnothing, K)$, and $(A, H)=$ auto-partial-reduce $\left(A_{N}, H_{N}\right)$.

We begin with an auxiliary result, which is an analogue of the first property from [7, Section 5.1].

LemMa 1. For every $f \in A_{i}$ and $i<j$, there exists $g \in A_{j}$ such that $\operatorname{lead}(f) \in \Theta \operatorname{lead}(g)$. In particular, if $p$ is reduced with respect to $A_{j}$, then $p$ is also reduced with respect to $A_{i}$ for all $i<j$.

Proof. It is sufficient to consider the case $j=i+1$. If $\left(G_{i+1}, D_{i+1}, A_{i+1}, H_{i+1}\right)$ was obtained from $\left(G_{i}, D_{i}, A_{i}, H_{i}\right)$ without applying the update operation, then $A_{i}=A_{i+1}$. Otherwise, either $f \in A_{i} \backslash G_{A_{i}}$ (we use the notation from the update algorithm), or $f \in G_{A_{i}}$. In the former case, $f \in A_{i+1}$ as well, so we can set $g=f$. In the latter case, $\operatorname{lead}(f) \in \Theta \operatorname{lead}(p)$, so we can set $g=p$.
We define a partial order $\preccurlyeq$ on the set of derivatives $\Theta Y$ as follows. For $u, v \in \Theta Y$, we say that $u \preccurlyeq v$ if there exists $\theta \in \Theta$ such that $\theta u=v$. Note that this implies that $u$ and $v$ are both derivatives of the same $y \in Y$.

Definition 20. An antichain sequence in $\Theta Y$ is a sequence of elements $S=\left\{s_{1}, s_{2}, \ldots\right\} \subseteq \Theta Y$ that are pairwise incomparable in this partial order.

Given a sequence $\left\{\left(G_{i}, D_{i}, A_{i}, H_{i}\right)\right\}_{i=0}^{N}$ as above (where $N=N_{(A, H)}$ for some regular differential system $(A, H)$ in the output of the Rosenfeld-Gröbner algorithm), we will construct an antichain sequence $S=\left\{s_{1}, s_{2}, \ldots\right\} \subseteq \Theta Y$ inductively going along the sequence $\left\{\left(G_{i}, D_{i}, A_{i}, H_{i}\right)\right\}$.

Suppose $S_{j-1}=\left\{s_{1}, \ldots, s_{j-1}\right\}$ has been constructed after considering $\left(G_{0}, D_{0}, A_{0}, H_{0}\right), \ldots,\left(G_{i-1}, D_{i-1}, A_{i-1}, H_{i-1}\right)$, where $S_{0}=\varnothing$. A 4 -tuple $\left(G_{i}, D_{i}, A_{i}, H_{i}\right)$ can be obtained from the tuple $\left(G_{i-1}, D_{i-1}, A_{i-1}, H_{i-1}\right)$ in two ways:

1. We did not perform the update operation. In this case, we do not append a new element to $S$.

2. We performed update with respect to some differential polynomial $p$. If there exists $s_{k} \in S_{j-1}$ such that $\operatorname{lead}(p) \leqslant s_{k}$, we do not append a new element to $S_{j-1}$. Otherwise, we let $s_{j}=\operatorname{lead}(p)$ and define $S_{j}=$ $\left\{s_{1}, \ldots, s_{j}\right\}$. In the latter case, we set $k_{j}=i$.

We also set $k_{0}=0$.

THEOREM 1. The sequence $\left\{s_{j}\right\}$ is an antichain sequence in $\Theta Y$ and, for all $j \geqslant 1$,

$$
\operatorname{ord}\left(s_{j}\right) \leqslant h f_{j},
$$

where $\left\{f_{j}\right\}$ is the Fibonacci sequence.

Proof. Let $i<j$. Assume that $s_{j} \succcurlyeq s_{i}$. Then, $p$ is not reduced with respect to $A_{k_{i}}$, which contradicts Lemma 1 . On the other hand, the case $s_{j} \preccurlyeq s_{i}$ is impossible by the construction of the sequence, so $\left\{s_{j}\right\}$ is an antichain sequence.

We denote by anti- $k_{i}$ the maximal $j \in \mathbb{Z}_{\geqslant 0}$ such that $k_{j} \leqslant i$. For all $i \geqslant 0$, let us set $j=$ anti- $k_{i}$ and prove by induction on $i$ that

1. $\mathcal{H}\left(\bigcup_{t=0}^{i}\left(G_{t} \cup D_{t} \cup H_{t}\right)\right) \leqslant h f_{j+1}$;

2. $\mathcal{H}\left(\bigcup_{t=0}^{i} A_{t}\right) \leqslant h f_{j}$

3. For all distinct elements of $\bigcup_{t=0}^{i} A_{t}$, the orders of the least common derivatives of their leaders do not exceed $h f_{j+1}$.

In the base case $i=0=k_{0}$, we have

$$
\mathcal{H}\left(G_{0} \cup D_{0} \cup H_{0}\right)=h=h f_{1}
$$

and

$$
\mathcal{H}\left(A_{0}\right)=\mathcal{H}(\varnothing)=0=h f_{0} .
$$

There are two distinct cases for $i+1$ :

1. Case $i+1<k_{j+1} \quad$ (so we have anti- $k_{i+1}=j$ ). Then, $\left(G_{i+1}, D_{i+1}, A_{i+1}, H_{i+1}\right)$ was obtained from $\left(G_{i}, D_{i}, A_{i}, H_{i}\right)$ in one of the following ways: 
(a) We did not perform the update operation. In this case, $A_{i+1}=A_{i}$ and

$$
\mathcal{H}\left(G_{i+1} \cup D_{i+1} \cup H_{i+1}\right) \leqslant \mathcal{H}\left(G_{i} \cup D_{i} \cup H_{i}\right) .
$$

(b) We performed the update operation with respect to a differential polynomial $p$ such that $\operatorname{lead}(f) \in$ $\Theta \operatorname{lead}(p)$ for some $f \in \bigcup_{t=0}^{i} A_{t}$. In this case,

$$
\mathcal{H}\left(A_{i+1}\right) \leqslant \mathcal{H}\left(\bigcup_{t=0}^{i} A_{t}\right) .
$$

Then, for all $g \in A_{t}(t \leqslant i)$,

$$
\operatorname{ord}(\Delta(p, g)) \leqslant \operatorname{ord}(\operatorname{lcd}(\operatorname{lead}(g), \operatorname{lead}(f))),
$$

which is bounded by $h f_{j+1}$ due to the third inductive hypothesis. Since $D_{i+1} \backslash D_{i}$ consists of some of these polynomials, $G_{i+1} \backslash G_{i} \subseteq A_{i}$, and $H_{i+1} \backslash H_{i}=$ $\{\operatorname{sep}(p), \operatorname{init}(p)\}$, then

$$
\mathcal{H}\left(G_{i+1} \cup D_{i+1} \cup H_{i+1}\right) \leqslant \mathcal{H}\left(G_{i} \cup D_{i} \cup H_{i}\right) .
$$

2. Case $i+1=k_{j+1}$ (so now anti- $k_{i+1}=j+1$ ). We performed the update operation with respect to a differential polynomial $p$, which is a result of reduction of some $\tilde{p} \in G_{i} \cup D_{i}$ with respect to $A_{i}$. Then

$$
\mathcal{H}\left(A_{i+1}\right) \leqslant \max \left(\mathcal{H}\left(A_{i}\right), \operatorname{ord}(p)\right) \leqslant h f_{j+1} .
$$

Moreover, for every $g \in \bigcup_{t=0}^{i} A_{t}$,

$$
\operatorname{ord}(\operatorname{lcd}(\operatorname{lead}(g), \operatorname{lead}(p))) \leqslant h f_{j}+h f_{j+1}=h f_{j+2} .
$$

Since $D_{i+1} \backslash D_{i}$ consists of some of these polynomials, $G_{i+1} \backslash G_{i} \subseteq A_{i}$, and $H_{i+1} \backslash H_{i}=\{\operatorname{sep}(p)$, init $(p)\}$, we have

$$
\begin{aligned}
\mathcal{H}\left(G_{i+1} \cup D_{i+1} \cup H_{i+1}\right) & \\
& \leqslant \max \left(\mathcal{H}\left(G_{i} \cup D_{i} \cup H_{i}\right), h f_{j+2}\right)=h f_{j+2} .
\end{aligned}
$$

Since ord $\left(s_{j}\right) \leqslant \mathcal{H}\left(A_{k_{j}}\right) \leqslant h f_{j}$, this completes the proof.

Let $\mathfrak{n}=\{1, \ldots, n\}$. The set of derivatives $\Theta Y$ is in oneto-one correspondence with the set $\mathbb{Z}_{\geqslant 0}^{m} \times \mathfrak{n}$ by associating $\partial_{1}^{i_{1}} \cdots \partial_{m}^{i_{m}} y_{k} \in \Theta Y$ to $\left(\left(i_{1}, \ldots, i_{m}\right), k\right) \in \mathbb{Z}_{\geqslant 0}^{m} \times \mathfrak{n}$. We define the degree of an element of $\mathbb{Z}_{\geqslant 0}^{m} \times \mathfrak{n}$ to be the order of its corresponding element of $\Theta Y$.

Under this correspondence, the partial order $\preccurlyeq$ on $\Theta Y$ determines a partial order $\preccurlyeq$ on $\mathbb{Z}_{\geqslant 0}^{m} \times \mathfrak{n}$ by saying $\left(\left(i_{1}, \ldots, i_{m}\right), k\right) \preccurlyeq\left(\left(j_{1}, \ldots, j_{m}\right), l\right)$ if and only if $k=l$ and $i_{r} \leqslant j_{r}$ for all $1 \leqslant r \leqslant m$. Thus there is a one-to-one correspondence between the set of antichain sequences of $\Theta Y$ and the set of antichain sequences of $\mathbb{Z}_{\geqslant 0}^{m} \times \mathfrak{n}$. Every antichain sequence of $\mathbb{Z}_{\geqslant 0}^{m} \times \mathfrak{n}$ (and thus of $\Theta Y$ ) is finite; see [12, Lemma 4.4].

Given an increasing function $f: \mathbb{Z}_{>0} \rightarrow \mathbb{Z}_{\geqslant 0}$, we say that $f$ bounds the degree growth of an antichain sequence $S=$ $\left\{s_{1}, \ldots, s_{k}\right\} \subseteq \mathbb{Z}_{\geqslant 0}^{m} \times \mathfrak{n}$ if $\operatorname{deg}\left(s_{i}\right) \leqslant f(i)$ for all $1 \leqslant i \leqslant k$. In $[12$, Lemma 4.9], it is shown that there is an upper bound on the length of an antichain sequence of $\mathbb{Z}_{\geqslant 0}^{m} \times \mathfrak{n}$ with degree growth bounded by $f$, and this upper bound depends solely on $m, n$, and $f$. We let $\mathfrak{L}_{f, m}^{n}$ be the maximal length of an antichain sequence of $\mathbb{Z}_{\geqslant 0}^{m} \times \mathfrak{n}$ with degree growth bounded by $f$.
TheOrem 2. Let $F, K \subseteq \boldsymbol{k}\{Y\}$ be finite subsets with $h=$ $\mathcal{H}(F \cup K)$, let $\mathcal{A}=$ Rosenfeld-Gröbner $(F, K)$, and let $L=$ $\mathfrak{L}_{f, m}^{n}$, where $f(i)=h f_{i}$ with $\left\{f_{i}\right\}$ the Fibonacci sequence. Then

$$
\mathcal{H}\left(\bigcup_{(A, H) \in \mathcal{A}}(A \cup H)\right) \leqslant h f_{L+1} .
$$

Proof. Since ord $(\operatorname{pd}-\operatorname{red}(p, B)) \leqslant \operatorname{ord}(p)$ for any $p \in$ $\mathbf{k}\{Y\}$ and weak d-triangular set $B$, this means that

$$
\mathcal{H}(B \cup K) \leqslant \mathcal{H}(A \cup H),
$$

where $\{(B, K)\}=$ auto-partial-reduced $(A, H)$. Hence, it suffices to bound $\mathcal{H}(G \cup D \cup A \cup H)$ whenever the tuple $(G, D, A, H)$ is added to $\mathcal{S}$ in the Rosenfeld-Gröbner algorithm.

By Theorem 1 and the correspondence between antichain sequences of $\Theta Y$ and $\mathbb{Z}_{\geqslant 0}^{m} \times \mathfrak{n}$, we obtain an antichain sequence of $\mathbb{Z}_{\geqslant 0}^{m} \times \mathfrak{n}$ of degree growth bounded by $f(i)$, so the length of this sequence (and thus the sequence from Theorem 1 ) is at most $L$.

In the proof of Theorem 1 , it is shown that for all $i \leqslant N$, for $j:=$ anti- $k_{i}$, we have

$$
\mathcal{H}\left(\bigcup_{t=1}^{i}\left(G_{t} \cup D_{t} \cup A_{t} \cup H_{t}\right)\right) \leqslant h f_{j+1} .
$$

Since the largest possible $j$ is the length of the antichain sequence (and this $j$ is equal to anti- $k_{N}$ ), for every $\left(G_{i}, D_{i}, A_{i}, H_{i}\right)$, we have

$$
\mathcal{H}\left(G_{i} \cup D_{i} \cup A_{i} \cup H_{i}\right) \leqslant h f_{L+1} .
$$

Since every $(G, D, A, H)$ added to $\mathcal{S}$ is equal to $\left(G_{i}, D_{i}, A_{i}, H_{i}\right)$ for some $i$, this completes the proof.

\section{SPECIFIC VALUES}

In order to apply Theorem 2, we need to be able to effectively compute $\mathfrak{L}_{f, m}^{n}$. [12] only proves the existence of this number, without an analysis of how to construct it. [4] constructs an upper bound for $m=1,2$. The first analysis for the case of arbitrary $m$ appears in [11].

Let $f: \mathbb{Z}_{>0} \rightarrow \mathbb{Z}_{\geqslant 0}$ be an increasing function. Let us define a function $\Psi_{f, m}: \mathbb{Z}_{>0} \times \mathbb{Z}_{\geqslant 0}^{m} \rightarrow \mathbb{Z}_{\geqslant 0}$ by the following relations:

$$
\begin{aligned}
& \Psi_{f, m}\left(i,\left(0, \ldots, 0, u_{m}\right)\right)=i, \\
& \quad \Psi_{f, m}\left(i-1,\left(u_{1}, \ldots, u_{r}, 0, \ldots, 0, u_{m}\right)\right) \\
& =\Psi_{f, m}\left(i,\left(u_{1}, \ldots, u_{r}-1, f(i)-f(i-1)+u_{m}+1,0, \ldots, 0\right)\right) \\
& \text { if } r<m-1, u_{r}>0, \text { and } \\
& \quad \Psi_{f, m}\left(i-1,\left(u_{1}, \ldots, u_{m}\right)\right) \\
& =\Psi_{f, m}\left(i,\left(u_{1}, \ldots, u_{m-1}-1, f(i)-f(i-1)+u_{m}+1\right)\right)
\end{aligned}
$$

if $u_{m-1}>0$.

Proposition 1 ([11, Corollary 3.10]).

The maximal length of an antichain sequence in $\mathbb{Z}_{\geqslant 0}^{m}$ with degree growth bounded by $f$ does not exceed

$$
\Psi_{f, m}(1,(f(1), 0, \ldots, 0)) .
$$


Let us also define the sequence $\psi_{0}, \psi_{1}, \ldots$ by the relations $\psi_{0}=0$ and

$$
\psi_{i+1}=\Psi_{f_{i}, m}\left(1,\left(f_{i}(1), 0, \ldots, 0\right)\right)+\psi_{i},
$$

where $f_{i}(x)=f\left(x+\psi_{i}\right)$.

Proposition 2 ([11, Corollary 3.14]). The maximal length of an antichain sequence in $\mathbb{Z}_{\geqslant 0}^{m} \times \mathfrak{n}$ with degree growth bounded by $f$ does not exceed $\psi_{n}$.

Now, let us apply this technique to the function $f(i)=$ $h f_{i}$. We then have, by Theorem 2, that an upper bound on the orders of the output of the Rosenfeld-Gröbner algorithm will be $f\left(\mathfrak{L}_{f, m}^{n}+1\right)$. In general, we cannot construct a formula for $\mathfrak{L}_{f, m}^{n}$ for arbitrary $h, m, n$ that improves upon the one given in Proposition 2; however, we can compute $\mathfrak{L}_{f, m}^{n}$ for some specific values of $h, m, n$.

1. Assume that $n=1$ and $m=2$. Then, the maximal length of an antichain sequence does not exceed $h+1$. In this case, the orders of the resulting polynomials are bounded by the single-exponential estimate, coming from the standard formula for the Fibonacci numbers,

$$
h f_{h+2}=\frac{h}{\sqrt{5}}\left(\left(\frac{1+\sqrt{5}}{2}\right)^{h+2}-\left(\frac{1-\sqrt{5}}{2}\right)^{h+2}\right) .
$$

This results in the following table:

\begin{tabular}{|c|c|c|c|c|c|c|c|}
\hline$h$ & 1 & 2 & 3 & 4 & 5 & 6 & 7 \\
\hline$h f_{h+2}$ & 2 & 6 & 15 & 32 & 65 & 126 & 238 \\
\hline
\end{tabular}

2. Assume that $m=2$ and $n$ is arbitrary. Then, the maximal length of an antichain sequence does not exceed $b_{n}$, where $b_{n}$ satisfies $b_{1}=h+1$ and $b_{n+1}=h f_{b_{n}+1}+b_{n}+1$, which results in the following table:

\begin{tabular}{|c|c|c|c|}
\hline$n$ & $h$ & $b_{n}$ & $h f_{b_{n}+1}$ \\
\hline 2 & 1 & 5 & 8 \\
\hline 2 & 2 & 10 & 178 \\
\hline 2 & 3 & 20 & 32,838 \\
\hline 2 & 4 & 38 & $252,983,944$ \\
\hline 2 & 5 & 72 & $\leq 10^{16}$ \\
\hline 3 & 1 & 14 & 610 \\
\hline 3 & 2 & 189 & $\leq 10^{40}$ \\
\hline 3 & 3 & 32,859 & $\leq 10^{6868}$ \\
\hline
\end{tabular}

3. Assume that $m=3$ and $n=1$. We can construct the maximal length antichain sequence of $\mathbb{Z}_{\geqslant 0}^{3}$ using the methods of [11], resulting in the following sequence:

$$
\begin{gathered}
(h, 0,0),(h-1,1,0),(h-1,0, h+1),(h-2,2 h+2,0), \ldots, \\
\left(h-2,0, h f_{2 h+6}-(h-2)\right), \ldots,\left(h-i, h f_{c_{i-1}+1}-(h-i), 0\right), \ldots, \\
\left(h-i, 0, h f_{c_{i}}-(h-i)\right), \ldots,\left(0, h f_{c_{h-1}+1}, 0\right), \ldots,\left(0,0, h f_{c_{h}}\right),
\end{gathered}
$$

where the sequence $c_{i}$ is given by $c_{0}=1$ and for $1 \leqslant i \leqslant h$,

$$
c_{i}=c_{i-1}+1+h f_{c_{i-1}+1}-(h-i) .
$$

As a result, we see that the maximal length of an antichain sequence is equal to $c_{h}$.

Below is a table of some maximal lengths $\mathfrak{L}_{f, m}^{n}$ and orders $f\left(\mathfrak{L}_{f, m}^{n}+1\right)$, where $f(i)=h f_{i}$, for $m=3,4$, and 5 :

\begin{tabular}{|c|c|c|c|c|}
\hline$m$ & $n$ & $h$ & length $\leqslant$ & order \\
\hline 3 & 1 & 1 & 3 & 3 \\
\hline 3 & 1 & 2 & 10 & 178 \\
\hline 3 & 1 & 3 & 712 & $10^{150}$ \\
\hline 3 & 2 & 1 & $433,494,480$ & $10^{90,594,990}$ \\
\hline 4 & 1 & 1 & 5 & 8 \\
\hline 5 & 1 & 1 & 20 & 10,946 \\
\hline
\end{tabular}

\section{ORDER LOWER BOUND}

This section gives a lower bound for the orders of the output of the Rosenfeld-Gröbner algorithm, coming from the lower bound for degrees of elements of a Gröbner basis from [14]. To be specific, we show that for $m, h$ sufficiently large, there is a collection of $r$ differential polynomials $F \subseteq \mathbf{k}\{y\}$ of order at most $h$, where $\mathbf{k}$ is equipped with $m$ derivations, $r \sim$ $m / 2$, and $\mathbf{k}$ is constant with respect to all of the derivations, such that if $\mathcal{A}=\operatorname{Rosenfeld-Gröbner}(F,\{1\})$, then

$$
\mathcal{H}\left(\bigcup_{(A, H) \in \mathcal{A}}(A \cup H)\right) \geqslant h^{2^{r}} .
$$

The arguments presented here are standard, and we include them for completeness. We first note the following standard fact about differential ideals generated by linear differential polynomials.

Proposition 3. Suppose $F, K \subseteq \boldsymbol{k}\{Y\}$ are composed of linear differential polynomials. Then the output of Rosenfeld-Gröbner $(F, K)$ is either empty or consists of a single regular differential system $(A, H)$ with $A$ and $H$ both composed of linear differential polynomials.

Suppose now we apply the Rosenfeld-Gröbner algorithm to $(F,\{1\})$, where $F$ consists of linear differential polynomials, in order to obtain a regular decomposition of $\{F\}$. Since every element of $F$ is linear, $[F]$ is a prime differential ideal, so by Proposition 3, we have

$$
[F]=\{F\}=[A]: H^{\infty}
$$

for some regular differential system $(A, H)$, with $A$ and $H$ both composed of linear differential polynomials. Since every element of $A$ is linear, after simply performing scalar multiplications and addition, $A$ can be transformed to an autoreduced set $\bar{A}$ without affecting the leaders and orders of elements of $A$. Since $(A, H)$ is a regular differential system, $\bar{A}$ is a characteristic set of $[F]$. As a result, it suffices to find a lower bound on the orders of elements of linear characteristic sets in $\mathbf{k}\{Y\}$.

There is a well-studied one-to-one correspondence between polynomials in $\mathbf{k}\left[x_{1}, \ldots, x_{m}\right]$ and homogeneous linear differential polynomials in $\mathbf{k}\{y\}$ with $m$ derivations and $\mathbf{k}$ a field of constants via

$$
\sum c_{i_{1}, \ldots, i_{m}} x_{1}^{i_{1}} \cdots x_{m}^{i_{m}} \leftrightarrow \sum c_{i_{1}, \ldots, i_{m}} \partial_{1}^{i_{1}} \cdots \partial_{m}^{i_{m}} y .
$$

Any orderly ranking on $\Theta y$ then determines a graded monomial order on $\mathbf{k}\left[x_{1}, \ldots, x_{m}\right]$.

Given a polynomial $f \in \mathbf{k}\left[x_{1}, \ldots, x_{m}\right]$, let $\tilde{f} \in \mathbf{k}\{y\}$ be its corresponding differential polynomial under (5). By the discussion above, if we have a collection of polynomials $f_{1}, \ldots, f_{r} \in \mathbf{k}\left[x_{1}, \ldots, x_{m}\right]$, we can construct a characteristic set $C=\left\{C_{1}, \ldots, C_{s}\right\}$ of $\left[\tilde{f}_{1}, \ldots, \tilde{f}_{r}\right] \subseteq \mathbf{k}\{y\}$ consisting of homogeneous linear differential polynomials, and so each $C_{i} \in \mathbf{k}\{y\}$ is in fact equal to $\tilde{g}_{i}$ for some $g_{i} \in \mathbf{k}\left[x_{1}, \ldots, x_{m}\right]$. 
Proposition 4 (CF. [13, PAGE 352],[5]).

With the notation above, $\left\{g_{1}, \ldots, g_{s}\right\} \subseteq \boldsymbol{k}\left[x_{1}, \ldots, x_{m}\right]$ is a Gröbner basis of the ideal $I=\left(f_{1}, \ldots, \bar{f}_{r}\right)$.

By Proposition 4, we can thus find a lower bound for the orders of the output of the Rosenfeld-Gröbner algorithm via a lower bound for the degrees of elements of a Gröbner basis, as we do in the following example.

Example 1. This example demonstrates the lower bound (4) for the orders of the output of the Rosenfeld-Gröbner algorithm. In [14, Section 8], for $m, h$ sufficiently large, a collection of $m$ algebraic polynomials $f_{1}, \ldots, f_{r}$ of degree at most $h$ in $m$ algebraic indeterminates, with $r \sim m / 2$, is constructed such that any Gröbner basis of $\left(f_{1}, \ldots, f_{r}\right)$ with respect to a graded monomial order has an element of degree at least $h^{2^{r}}$.

As a result of the previous discussion, we have a collection of differential polynomials $F=\tilde{f}_{1}, \ldots, \tilde{f}_{r} \in \mathbf{k}\{y\}$ of order $h$ with $m$ derivations such that any linear characteristic set of $\left[\tilde{f}_{1}, \ldots, \tilde{f}_{r}\right]$ will contain a differential polynomial of order at least $h^{2^{r}}$. Since in this case $\{(A, H)\}=$ Rosenfeld-Gröbner $(F,\{1\})$ can be transformed into a linear characteristic set without affecting the orders of the elements, this means that

$$
\mathcal{H}(A \cup H) \geqslant h^{2^{r}} .
$$

\section{Acknowledgments}

This work was supported by NSF grant CCF-0952591. We are grateful to the referees for their helpful comments and suggestions.

\section{References}

[1] F. Boulier, D. Lazard, F. Ollivier, and M. Petitot. Representation for the radical of a finitely generated differential ideal. In ISSAC'95: Proceedings of the $1995 \mathrm{in}$ ternational symposium on Symbolic and algebraic computation, pages 158-166, New York, NY, USA, 1995. ACM Press. URL http://dx.doi.org/10.1145/220346. 220367.

[2] F. Boulier, D. Lazard, F. Ollivier, and M. Petitot. Computing representations for radicals of finitely generated differential ideals. Applicable Algebra in Engineering, Communication and Computing, 20(1):73-121, 2009. URL http://dx.doi.org/10.1007/s00200-009-0091-7.

[3] L. D'Alfonso, G. Jeronimo, and P. Solernó. Effective differential Nullstellensatz for ordinary DAE systems with constant coefficients. Journal of Complexity, 30 (5):588-603, 2014. URL http://dx.doi.org/10.1016/j. jco.2014.01.001.

[4] J. Freitag and O. León Sánchez. Effective uniform bounds in partial differential fields. Advances in Mathematics, 288:308-336, 2016. URL http://dx.doi.org/10. 1016/j.aim.2015.10.013.
[5] V. Gerdt. Involutive algorithms for computing Gröbner bases. In Computational commutative and noncommutative algebraic geometry, volume 196 of NATO Science Series III: Computer and Systems Sciences. IOS, Amsterdam, 2005. URL http://arxiv.org/abs/ math/0501111.

[6] O. Golubitsky, M. Kondratieva, M. Moreno Maza, and A. Ovchinnikov. A bound for Rosenfeld-Gröbner algorithm. Journal of Symbolic Computation, 43(8):582610, 2008. URL http://dx.doi.org/10.1016/j.jsc.2007. 12.002 .

[7] O. Golubitsky, M. Kondratieva, A. Ovchinnikov, and A. Szanto. A bound for orders in differential Nullstellensatz. Journal of Algebra, 322(11):3852-3877, 2009. URL http://dx.doi.org/10.1016/j.jalgebra.2009.05.032.

[8] R. Gustavson, M. Kondratieva, and A. Ovchinnikov. New effective differential Nullstellensatz. Advances in Mathematics, 290:1138-1158, 2016. URL http://dx.doi. org/10.1016/j.aim.2015.12.021.

[9] E. Hubert. Notes on triangular sets and triangulationdecomposition algorithms II: Differential systems. In U. Langer and F. Winkler, editors, Symbolic and $\mathrm{Nu}$ merical Scientific Computations, volume 2630 of Lecture Notes in Computer Science, pages 40-87. Springer, 2003. URL http://dx.doi.org/10.1007/3-540-45084-X_ 2 .

[10] E. R. Kolchin. Differential algebra and algebraic groups. Academic Press, New York, 1973. Pure and Applied Mathematics, Vol. 54.

[11] O. León Sánchez and A. Ovchinnikov. On bounds for the effective differential Nullstellensatz. Journal of Algebra, 449:1-21, 2016. URL http://dx.doi.org/10.1016/ j.jalgebra.2015.10.009.

[12] D. Pierce. Fields with several commuting derivations. Journal of Symbolic Logic, 79(1):1-19, 2014. URL http: //dx.doi.org/10.1017/jsl.2013.19.

[13] W. T. Wu. On the construction of Groebner basis of a polynomial ideal based on Riquier-Janet theory. In D. Wang and Z. Zheng, editors, Differential Equations with Symbolic Computation, Trends in Mathematics, pages 351-368. Birkhäuser Verlag, 2005. URL http://dx.doi.org/10.1007/3-7643-7429-2_20.

[14] C. Yap. A new lower bound construction for commutative Thue systems with applications. Journal of Symbolic Computation, 12:1-27, 1991. URL http: //dx.doi.org/10.1016/S0747-7171(08)80138-1. 